\title{
A Clinical and Radiographic Study of Four Different Root Canal Filling Materials in Primary Molars- An In Vivo Study
}

\author{
Bhavleen Gupta ${ }^{1}$ Inderjeet Singh ${ }^{1} \quad$ Puneet Goyal ${ }^{1}$ Shaveta Garg ${ }^{1}$ Shivani Gupta ${ }^{1}$ \\ ${ }^{1}$ Department of Pediatric \& Preventive Dentistry, Guru Nanak Dev \\ Address for correspondence Puneet Goyal, MDS, Department of \\ Pediatric \& Preventive Dentistry, Guru Nanak Dev Dental College \& \\ Research Institute, Sunam, Patiala 148028, Punjab, India \\ (e-mail: go2goyal@gmail.com).
}

Dent J Adv Stud 2019;7:61-65

\begin{abstract}
Keywords

- Endoflas

- Metapex

- zinc oxide propolis

- zinc oxide eugenol
\end{abstract}

Introduction Pulpectomy helps in preserving a pulpally involved primary tooth by eliminating bacteria and their products and ensures hermetic seal of the root canals, so that the primary teeth can complete their function until normal exfoliation. For the success of endodontic treatment in primary teeth, the resorption of obturating material plays a vital role. The materials used for obturation in this study included zinc oxide propolis (ZOP) as a new paste, Endoflas, Metapex, and zinc oxide eugenol (ZOE). Objective To evaluate the success rate of pulpectomy of primary molars using four different root canal filling materials: ZOP as a new paste, Endoflas, Metapex, and ZOE. Materials and Methods A total of 48 primary molars were divided into four equal groups of 12 molars according to the type of root canal filling paste, that is, ZOE, Endoflas, ZOP, and Metapex.

Results When Tukey's test was applied, out of the four materials, ZOP was the most significant material as its $p$-value was $<0.05$, whereas other materials showed nonsignificant results as their $p$-value was $>0.05$.

Conclusion Among the four groups, ZOP showed greater clinical success and its resorbability was similar to that of root.

\section{Introduction}

Objective of pulp therapy in case of deciduous teeth is to maintain the teeth in cases in which the pulpal tissue gets affected by caries exposure, trauma, or other causes, disrupting the functional state until replaced by succedaneous permanent teeth. Irreversibly infected or necrotic pulpal tissue requires pulpectomy as the treatment of choice. To increase the chances of success of the endodontic treatment, materials with antimicrobial properties are frequently used as obturating material in deciduous teeth. ${ }^{1}$

The main purpose of endodontic treatment is the complete elimination of microorganisms from the root canal and its prevention from subsequent reinfection that can be achieved by careful cleaning and shaping, followed by the complete obturation of the canal space. ${ }^{2}$

received

February 20, 2019

accepted after revision

April 26, 2019

published online

June 14, 2019
Zinc oxide eugenol (ZOE), a widely used root canal filling material for deciduous teeth, was cited as the preferred root canal filling material by $94 \%$ chairpersons of predoctoral pediatric dental programs in United States in 1997. However, owing to limited antimicrobial action and slower resorption rate, ZOE cannot be regarded as an ideal root canal filling material.

Iodoform paste, a better disinfectant of the root canal with better resorbability, produces a yellowish-brown discoloration of the tooth crowns, which undermines esthetics. ${ }^{1}$ The foremost disadvantage of calcium hydroxide is its tendency to resorb earlier than the physiologic resorption of root of the primary teeth, which creates a "hollow tube" effect.

Endoflas paste has the merit of having resorption confined to the excess extruded material and remains intact within the canal. Thus, the material is neither resistant to resorption nor does it result in the hollow tube

(C)2019 Bhojia Dental College and Hospital affiliated to Himachal

Pradesh University
License terms

() (1) $\ominus \circledast$
$10.1055 / \mathrm{s}-0039-1692611$

ISSN 2321-1482. 
effect. Its hydrophilic properties make it useful for humid canals. It has the ability to disinfect dentinal tubules and hard-to-reach accessory canals that cannot be disinfected or cleansed mechanically. ${ }^{3}$ Propolis, a nontoxic resinous natural substance with antimicrobial, anticancer, antifungal, antiviral, and anti-inflammatory attributes, has gained attention in dental as well as medical arena. Having its origin from a Greek words pro (making outer wall) and polis (meaning city), propolis is a natural product of bees and is used for building and preservation of their hives. ${ }^{4}$

\section{Selection Criteria}

A total of 48 primary teeth root canals in healthy, cooperative children aged 4 to 8 years (either male or female) having at least 1 primary tooth indicated for pulpectomy were selected. Teeth were randomly divided into four groups: group I: zinc oxide propolis (ZOP) as a new paste (K-Link healthcare, Selangor, Malaysia), group II: Endoflas (Sanlor Laboratories, Miami, Florida, United States), group III: Metapex paste (Meta Biomed, Korea), and group IV: ZOE paste as a control paste (DPI, Mumbai, Maharastra, India).

\section{Inclusion Criteria}

1. Age group: 4 to 8 years

2. Acute pulpitis

3. Chronic pulpitis

4. Carious teeth with sinus formation

5. Pulp necrosis

6. Primary teeth with carious pulp exposure in which, following coronal pulp amputation, the radicular pulp exhibits clinical signs of hyperemia

7. Primary teeth with at least two-thirds of root should be present

\section{Exclusion Criteria}

1. Unrestorable tooth

2. Teeth with excessive pathological mobility

3. Medically compromised children

4. Teeth showing sign and symptoms of pathologic internal and external resorption

\section{Methodology}

Overall 48 primary teeth root canals in cooperative children were randomly divided into four groups.

A single material among the specified four materials was used for filling of root canals. The endodontically treated tooth was finally restored permanently with stainless steel crown (-Table 1).

\section{Criteria for Clinical Evaluation at 3 and $\mathbf{6}$ Months}

Presence or absence of pain, swelling or abscess, draining fistula, mobility.

\section{Criteria for Radiographic Findings at 3 and $\mathbf{6}$ Months}

Presence or absence of furcation radiolucency, abnormal root resorption, internal root resorption, external root resorption, excess filling material and its resorption.

\section{Results}

When one-way ANOVA (analysis of variance) test was applied, ZOP had the greatest mean value, that is, 94.50, whereas ZOE had the least mean value, that is, 83.92 , but results were found to be significant as $p$-value was $<0.05$ ( - Table 2).

\section{Discussion}

The principal aim of endodontic therapy is to maintain the integrity and function of teeth and their supporting tissues. The pulpectomy procedure is the treatment of choice in case of irreversibly infected pulpal tissue. ${ }^{5}$

Endodontic procedure not only aids in preserving pulpally involved primary tooth by eradicating bacteria and their products but also ensures a hermetic seal of the root canals. ${ }^{6}$

Discrete materials used for obturation in primary teeth include ZOE, calcium hydroxide, Walkhoffs paste, KRI paste, Maisto paste, Vitapex, and Endoflas. ${ }^{7}$ Accomplishment of an endodontic treatment is marked by the eradication of the microorganisms and its products from the root canal. The materials used for obturation were ZOE, Endoflas, Metapex, and ZOP.

\section{Zinc Oxide Eugenol}

ZOE cement is one among the oldest known root canal filling material for deciduous teeth.

Anti-inflammatory and analgesic properties, greater zone of bacterial inhibition, ease of availability, radiopacity of material, cheaper, insolubility in tissue fluids, easy to mix, and good working time are the advantages of ZOE. ${ }^{8}$

Disadvantages include necrosis of cementum and bone, variation in resorption rate of ZOE, or slow resorption in comparison to root resorption. ${ }^{8}$

In a study by Mortazavi and Mesbahi, ${ }^{9}$ it was found that in comparison with the success rate of ZOE and Vitapex, the overall success rates were found to be $100 \%$ and $78.5 \%$, respectively.

In this study, when ZOE was used, five cases (two at 3 months and three at 6 months follow-up) were reported with pain. Also, four cases (two at 3 months and one at 6 months follow-up) were reported with swelling. A few cases (two at 3 months and one at 6 months follow-up) were reported with draining fistula. These clinical observations in the ZOE group were not found statistically significant as compared with the other groups. Saxena et al, ${ }^{6}$ in their study, suggested that the lowest success rate of 
Table 1 Clinical and radiographic evaluation of obturating materials at 6 months follow-up

\begin{tabular}{|c|c|c|c|}
\hline \multirow[t]{2}{*}{ Parameters at 6 months follow-up } & \multirow[t]{2}{*}{ Filling paste } & \multicolumn{2}{|l|}{ Index } \\
\hline & & Success (\%) & Failure (\%) \\
\hline \multirow[t]{4}{*}{ Pain } & ZOP & 92 & 8 \\
\hline & Endoflas & 92 & 8 \\
\hline & Metapex & 92 & 8 \\
\hline & ZOE & 75 & 25 \\
\hline \multirow[t]{4}{*}{ Swelling } & ZOP & 92 & 8 \\
\hline & Endoflas & 92 & 8 \\
\hline & Metapex & 100 & 0 \\
\hline & $\mathrm{ZOE}$ & 92 & 8 \\
\hline \multirow[t]{4}{*}{ Draining fistula } & ZOP & 92 & 8 \\
\hline & Endoflas & 92 & 8 \\
\hline & Metapex & 92 & 8 \\
\hline & ZOE & 75 & 25 \\
\hline \multirow[t]{4}{*}{ Mobility } & ZOP & 92 & 8 \\
\hline & Endoflas & 92 & 8 \\
\hline & METAPEX & 100 & 0 \\
\hline & ZOE & 75 & 25 \\
\hline \multirow[t]{4}{*}{ Furcation radiolucency } & ZOP & 100 & 0 \\
\hline & Endoflas & 100 & 0 \\
\hline & Metapex & 100 & 0 \\
\hline & $\mathrm{ZOE}$ & 92 & 8 \\
\hline \multirow[t]{4}{*}{ Root resorption } & ZOP & 100 & 0 \\
\hline & Endoflas & 83 & 17 \\
\hline & Metapex & 17 & 83 \\
\hline & ZOE & 50 & 50 \\
\hline \multirow[t]{4}{*}{ Excess filling } & ZOP & 0 & 0 \\
\hline & Endoflas & 50 & 50 \\
\hline & Metapex & 100 & 0 \\
\hline & ZOE & $0 \%$ & 0 \\
\hline
\end{tabular}

Abbreviations: ZOE, zinc oxide eugenol; ZOP, zinc oxide propolis.

Table 2 Comparison of success percentage on the basis of one-way ANOVA test

\begin{tabular}{|l|l|l|l|l|l|}
\hline Comparing group & Mean & SD & F-value & $p$-Value & Status \\
\cline { 1 - 3 } ZOP & 97.33 & 3.94 & 3.153 & 0.034 & Significant \\
\cline { 1 - 3 } Endoflas & 94.56 & 6.44 & & \\
\cline { 1 - 4 } & 85.50 & 28.69 & & & \\
\hline
\end{tabular}

Abbreviations: ANOVA, analysis of variance; SD, standard deviation; ZOE, zinc oxide eugenol; ZOP, zinc oxide propolis.

ZOE paste may be because ZO powder has no significant antibacterial properties, lack of acidity, and lack of release of antibacterial agents.

Various deficiencies that lead to exploration of alternative root canal filling materials are the limited antimicrobial action and resorption at slower rate. After crossing the apex, it causes a risk of deflection in succedaneous teeth due to its hardness. ${ }^{1}$

\section{Metapex}

Easy resorption from the periapical areas, no foreign-body reaction, and potent germicidal properties are the requirements fulfilled by iodoform pastes that can successfully be used as root canal filling material. If pushed beyond the apex, these materials show a resorption rate faster than the root. ${ }^{1}$ 
In a study by Mortazavi and Mesbahi, ${ }^{9}$ it was found that in comparison with ZOE and Vitapex, the comprehensive success rates of Vitapex and ZOE were found to be $100 \%$ and $78.5 \%$, respectively.

The efficacy of calcium hydroxide and ZOE as studied by Dogra, ${ }^{10}$ by using endodontic pressure syringe system, concluded that ZOE can be used as an alternative with calcium hydroxide as a root canal filling material.

In this study, when iodoform was used as obturating material and followed up for 3 months, it was seen that one patient reported with pain. In cases of overfilled canals, during follow-up complete resorption of material in periapical region was seen by the end of 6 months.

In a study conducted by Trairatvorakul and Chunlasikaiwan, ${ }^{11}$ the clinical and radiographic success rates of ZOE with Vitapex were compared. At 6 and 12 months, the success rates of ZOE were $48 \%$ and $85 \%$, respectively, and success rates of Vitapex were $78 \%$ and $89 \%$, respectively. It was seen that Vitapex appeared to resolve furcation pathology at a rate faster than ZOE. In this study, during 3- and 6-months follow-up, no case was reported with furcation radiolucency with Metapex, but one case of furcation radiolucency was reported when ZOE was used.

One of the detrimental properties of calcium hydroxide is that it has a tendency to resorb earlier than the physiologic resorption of root. This creates a "hollow tube" effect leading to an unfilled root that eventually becomes a site for infection. ${ }^{3}$

In this study, six cases were seen with excess filling material, and out of those, four cases showed resorption of excess filling material at 3 months and complete resorption at 6 months.

Vitapex resorbs faster than root. In contrast to ZOE, Vitapex leaves no residue in the tissue.

\section{Endoflas}

Endoflas paste was obtained by mixing a powder containing triiodomethane, zinc oxide, calcium hydroxide, barium sulphate, and iodine dibutilorthocresol, with a liquid containing eugenol and para-monochlorophenol. It has the advantage of having the resorption limited to the excess material, which has been extruded. Resorption of the material does not occur within the canal. Thus, the material is neither resistant to resorption nor does it result in the hollow tube effect. The material is hydrophilic and can be used in mild humid canals. It has the ability to disinfect dentinal tubules and hard to-reach accessory canals that cannot be disinfected or cleansed mechanically.

Rewal et $\mathrm{al}^{3}$ indicated that Endoflas with a success rate of $100 \%$ is a superior material compared to ZOE.

Ramar and Mungra ${ }^{12}$ compared the clinical and radiographic evaluation of Metapex, RC fill, and Endoflas for a duration of 9 months. Results showed that Endoflas gave an overall success rate of $95.1 \%$, Metapex $90.5 \%$, and RC Fill 84.7\%.

Fuks et $\mathrm{al}^{13}$ studied the success rate of Endoflas as a filling material in 55 primary teeth. Thirty-one teeth were overfilled, and of these, 29\% were normal preoperatively and the remaining $71 \%$ presented with bone pathology. Twenty-four teeth were flush or underfilled, and of these, $50 \%$ had preoperative bone pathology after 52 months. Seventy percent of the cases were successful, and the remaining $30 \%$ presented with pathology and only one tooth had to be extracted. Overfilling led to a success rate of $58 \%$, whereas in the combined flush and underfilled, the success rate was $83 \%$.

In this study, two cases showed excess filling material with Endoflas, and by the end of 6 months, only one case showed resorption of excess filling material. The time taken for the resorption of inadvertently extruded Endoflas has varied between 20-day and 11-month period in this study.

\section{Zinc Oxide Propolis}

Propolis, a potent resinous material, shows properties such as antimicrobial, anticancer, antifungal, antiviral, and antiinflammatory. ${ }^{7}$

Alafandy ${ }^{14}$ assessed the potency of crude propolis as a direct pulp capping agent in both primary and immature permanent teeth along with aesthetic and bone regenerating properties. On clinical and radiographic evaluation done after 1, 6, and 12 months, the results revealed that Syria crude propolis to be effective as a direct pulp-capping agent in immature permanent teeth.

In this study at 3 months follow-up, no case was reported with pain, swelling, draining fistula, and mobility when ZOP was used, but 1 case out of 12 reported with pain, swelling, draining fistula, and mobility at 6 months.

By comparing all the parameters, ZOP seems to be the best obturating material among all the four obturating material as results were statistically significant.

\section{Conflict of Interest}

None declared.

\section{References}

1 Barja-Fidalgo F, Moutinho-Ribeiro M, Oliveira MA, Oliveira BH. A systematic review of root canal filling materials for deciduous teeth: is there an alternative for zinc oxide-eugenol? ISRN Dent 2011;:1-7

2 Praveen P, Anantharaj A, Venkataragahavan K, Rani P, Sudhir R, Jaya AR. A review of obturating materials for primary teeth. SRM Univ J Dent Sci 2011;1(1):1-3

3 Rewal N, Thakur AS, Sachdev V, Mahajan N. Comparison of Endoflas and zinc oxide eugenol as root canal filling materials in primary dentition. J Indian Soc Pedod Prev Dent 2014;32(4):317-321

4 Khurshid Z, Naseem M, Zafar MS, Najeeb S, Zohaib S. Propolis: a natural biomaterial for dental and oral healthcare. J Dent Res Dent Clin Dent Prospect 2017;11(4):265-274

5 Barr ES, Kleier DJ, Barr NV. Use of nickel-titanium rotary files for root canal preparation in primary teeth. Pediatr Dent 2000;22(1):77-78

6 Saxena A, Koul M, Grover SD. An in vivo evaluation of different root canal filling materials used in primary teeth. Int J Oral Care Res 2017;5(3):196-201 
7 Bhatia R, Nalik S, Singh S, Gupta N, Naik S. Periapical and. intraradicular. resorption of extruded Endoflas in primary molar: a case report. A Publication of Indian Endodontic Society 2012;24(2):156-159

8 Chonat A, Rajamani T, Ephraim R. Obturating materials in primary teeth-a review, research and reviews. J Dent Sci 2018;6(1):20-25

9 Mortazavi M, Mesbahi M. Comparison of zinc oxide and eugenol, and Vitapex for root canal treatment of necrotic primary teeth. Int J Paediatr Dent 2004;14(6):417-424

10 Dogra S. Comparative evaluation of calcium hydroxide and zinc oxide eugenol as root canal filling materials for primary molars: a clinical and radiographic study, world. J Dent 2011;2(3):231-236
11 Trairatvorakul C, Chunlasikaiwan S. Success of pulpectomy with zinc oxide-eugenol vs calcium hydroxide/iodoform paste in primary molars: a clinical study. Pediatr Dent 2008;30(4):303-308

12 Ramar K, Mungara J. Clinical and radiographic evaluation of pulpectomies using three root canal filling materials: an in-vivo study. J Indian Soc Pedod Prev Dent 2010;28(1):25-29

13 Fuks AB, Eidelman E, Pauker N. Root fillings with Endoflas in primary teeth: a retrospective study. J Clin Pediatr Dent 2002;27(1):41-45

14 Alafandy AS. Assessment of crude propolis as a direct pulp capping agent in primary and immature permanent teeth. Endodontic Practice Today 2014;8(3):199-206 